\section{Redes y medios de transporte en el desarrollo de expediciones científicas en Argentina (1850-1910)}

\section{Transportation networks and means in the development of scientific expeditions in Argentina (1850-1910)}

FARRO, Máximo E. Redes y medios de transporte en el desarrollo de expediciones científicas en Argentina (1850-1910). História, Ciências, Saúde Manguinhos, Rio de Janeiro, v.15, n.3, p.679-696, jul.-set. 2008.

\section{Resumen}

Examina la relación entre el avance en las tecnologías del transporte y el desarrollo de las expediciones de carácter científico entre fines del siglo XIX y principios del siglo XX en Argentina. Se muestra cómo la expansión a escala nacional de la red de ferrocarriles impactó en el desarrollo de las prácticas en el terreno, ampliando el acceso a sitios distantes de las instituciones científicas ubicadas en Córdoba, Buenos Aires y La Plata, simplificando el movimiento de equipamiento, colecciones y personal. Se toman como ejemplo las expedicións realizadas por Hermann Burmeister (1857-1860), por los científicos de la Academia Nacional de Ciencias de Córdoba y las exploraciones arqueológicas en las provincias del noroeste organizadas por instituciones científicas con sede en Buenos Aires y La Plata.

Palabras clave: ciencias del terreno; redes y medios de transporte; logística del trabajo de campo; ferrocarriles; Argentina.

\section{Abstract}

The article explores the relation between the advance of transportation technology and the development of scientific expeditions between the late nineteenth and early twentieth centuries in Argentina. Expansion of the railway network on a national scale impacted the development of the earth sciences by facilitating access to distant places by scientific institutions located in Córdoba, Buenos Aires, and La Plata and also by simplifying the movement of equipment, scientific collections, and personnel. Hermann Burmeister's expedition (1857-60), expeditions by scientists from the Academia Nacional de Ciencias de Córdoba, and archeological digs in the northwestern provinces, organized by scientific institutions headquartered in Buenos Aires and La Plata, serve as examples.

Keywords: field sciences; transportation networks and means; fieldwork logistics, railroads; Argentina. 
$\mathrm{E}$ n los últimos años, el trabajo de campo ha sido definido y estudiado por los historiadores de la ciencia como una actividad práctica compleja, de naturaleza colectiva (Camerini, 1996; Kohler, 2002) que exige, para llevarse a cabo, la cooperación de un grupo socialmente diverso de practicantes, compuesto por los científicos, los directores de las instituciones, el personal de las reparticiones estatales, de los cuerpos militares y los residentes locales (Camerini, 1997; Rozwadowski, 1996) que le atribuyen distintos significados a los objetos recolectados y a las tareas llevadas a cabo en el terreno (Star, Griesemer, 1989). A menudo, estos informantes locales formaban parte de redes de corresponsales que colaboraban de distintas maneras con las instituciones científicas metropolitanas, intercambiando observaciones y especimenes, comunicando el hallazgo de objetos, recolectando observaciones meteorológicas o asistiendo al personal de aquellas por medio de la movilización de recursos locales, en forma de peones, baqueanos, medios de transporte, dinero, cartas de introducción, espacios de trabajo y almacenamiento de colecciones (Secord, 1994; Goldstein, 1994).

Por otro lado, Kuklick y Kohler (1996) han propuesto que el 'campo' o terreno puede ser estudiado en sí mismo, esto es, considerando la dimensión material de las tareas desarrolladas en ese medio.. Así, las prácticas en el terreno estarían determinadas en gran medida por las condiciones específicas de los lugares donde se llevan a cabo y para estudiarlas es necesario tener en cuenta la infraestructura disponible en aquellos lugares para el desarrollo de los trabajos, así como el equipamiento y la organización general de las tareas (Camerini, 1996, 1997). Un aspecto importante en esta línea de indagación es el estudio del desarrollo de las redes de transporte y comunicación, asociadas a la empresa científica, que hicieron posible la movilización de recursos, personal y colecciones de objetos y datos entre las instituciones dedicadas a la ciencia y las regiones a explorar (Podgorny, 2002, 2005; Podgorny, Schäffner, 2000).

En relación con esto, es de destacar que el 'campo' siempre se halla crucialmente situado dentro de una red de transporte y comunicación: por más próximo que se encuentre a su lugar de residencia, el trabajador de campo y sus asistentes deben tener un modo de llegar y volver de allí. Si bien los medios de transporte son importantes para el desarrollo de cualquier empresa científica, lo son más aún para el trabajo sobre el terreno. Contrariamente al científico que trabaja en el laboratorio, y que depende de las redes de transporte y comunicación a larga distancia - principalmente para la obtención de instrumental, reactivos o el intercambio de información en forma de protocolos y papers -, el investigador de campo ha dependido desde siempre de la existencia de animales de tiro, carretas, diligencias, barcos y botes, ferrocarriles o, ya en el siglo XX, el automóvil y el avión, para 'acceder' a su lugar de trabajo (Vetter, 2004, p.189).

Este trabajo pretende colaborar con los estudios que entienden el territorio a partir de la articulación de saberes científicos con las tecnologías del transporte y la comunicación. En este sentido, más que indagar, por un lado, en las características físicas del territorio, entendido como escollo natural a salvar, y por otro, en la preparación técnica de los estudiosos viajeros, nos interesa analizar la articulación entre la creación de una infraestructura para la transmisión y transporte de información, objetos y personas, y su influencia en el desarrollo de las expediciones científicas. Siguiendo estas ideas, en las 
páginas que siguen se muestra cómo la expansión de la red de ferrocarriles en el último tercio del siglo XIX impactó en el desarrollo de las prácticas en el terreno, facilitando tanto el acceso al campo mediante la reducción del tiempo de viaje, como la movilidad de los estudiosos y las colecciones.

\section{El acceso al terreno (1771-1870): los animales de tiro, el sistema de postas y mensajerías y los primeros vapores fluviales y marítimos}

Para comprender de manera más acabada el impacto que tuvo en el río de La Plata la introducción de sistemas de transporte y la comunicación basados en el vapor sobre el desarrollo de las exploraciones científicas durante el último tercio del siglo XIX y principios del XX, es importante describir brevemente las condiciones de desarrollo de las mismas en la etapa previa a los ferrocarriles y los barcos a vapor. En el caso del río de La Plata, el sistema de transporte implementado durante la época hispánica siguió funcionando, prácticamente sin variantes, al menos hasta mediados del siglo XIX (Cárcano, 1893; Castro Estévez, 1934-1942; Bose, 1966). El transporte de carga y de pasajeros por tierra se realizaba utilizando pesados carros de dos ruedas, tirados por bueyes o caballos, sobre caminos carreteros que, partiendo desde Buenos Aires, se internaban hacia el interior y seguían dos recorridos principales. Uno de ellos, conocido como la 'ruta del norte', partía hacia Pergamino; desde allí un ramal se dirigía a Rosario, Santa Fe, Corrientes y finalmente Asunción del Paraguay, y el otro continuaba hacia Córdoba y de allí, por Santiago del Estero, a Tucumán, Salta, Jujuy, Bolivia y Perú (Barba, 1956). La llamada 'ruta del sud' partía hacia San Luis y Mendoza, con una ruta complementaria menor que unía a esta última con San Juan (Bose, 1966; Jewell, 1966). Como muestra el Cuadro 1, comparada con los avances que se sucedieron en las décadas siguientes, la marcha en estas carretas era lenta. Movidas generalmente por tres o cuatro yuntas de bueyes, las carretas alcanzaban una velocidad del orden de los dos a cuatro kilómetros por hora: se recorría en promedio entre 15 y 35km por día; de esta manera, para llegar de Buenos Aires a Salta se demoraba entre sesenta y setenta días. Una forma más rápida de viajar era mediante el alquiler de caballos, utilizando el sistema de postas o 'camino del correo'. El origen del mismo se remonta a fines del siglo XVIII, cuando Don Alonso Carrió de la Vandera arribó al Plata como visitador de correos y postas con la misión de establecer las segundas en la ruta entre Buenos Aires y el Cuzco (Cárcano, 1893; Bose, 1966). Las postas eran lugares de relevo de la caballada y se ubicaban por lo general cerca de una fuente de agua potable y pasturas para los animales, a una distancia promedio, una de otra, de cuatro o cinco leguas (16-25km). Eran administradas por un 'maestro de posta', encargado de suministrar al viajero no sólo los animales necesarios para continuar la travesía, sino también el alojamiento y el refrigerio. Asimismo, era el responsable de la conducción del correo, tanto de los 'correos de número' como de aquél comisionado por el gobierno.

Todo viajero que utilizara este sistema debía llevar consigo un pasaporte extendido por autoridad competente, acompañado del 'parte' u hoja de ruta expedidos por el administrador de Correos. El pasaporte servía para controlar la circulación de personas por el país; en el parte adjunto al mismo, cada maestro de posta anotaba la fecha y hora 
de llegada y salida de los viajeros y su nuevo destino. Este sistema de postas, que partía hacia el sur de la ciudad de Buenos Aires hasta el fuerte de Carmen de Patagones, fue utilizado por Charles Darwin para estudiar en detalle las características geológicas de la formación pampeana y recolectar muestras geológicas y fósiles. Partiendo desde la boca del río Negro, el naturalista alcanzó el 8 de septiembre de 1833 el fuerte de bahía Blanca y desde allí, munido de un salvoconducto redactado por Juan Manuel de Rosas y escoltado por sus tropas, arribó a Buenos Aires el 20 del mismo mes, habiendo parado previamente en las postas de sierra de la Ventana, río Sauce, arroyo Tapalqué y Guardia del Monte (Podgorny et al., 2008).

Cuadro 1

Principales características de los medios de transporte terrestre a mediados del siglo XIX

\begin{tabular}{lccc}
\hline Medios & $\begin{array}{c}\text { Velocidad } \\
(\mathbf{k m} / \mathbf{h})\end{array}$ & $\begin{array}{c}\text { Recorrido diario } \\
\mathbf{( k m )}\end{array}$ & $\begin{array}{c}\text { Volumen de carga } \\
\text { (en tn ó pasajeros) }\end{array}$ \\
\hline Carreta & $3-4$ & $15-40$ & $1,5-2,2$ \\
Carro pesado & $2-3$ & $30-35$ & $3-5$ \\
Arrias & 6 & 60 & 0,150 a 0,175 \\
Diligencias & $13-16$ & $60-100$ & Hasta 12 pasajeros \\
Galeras & 20 & 150 & $14-17$ pasajeros \\
Caballos de chasquis & - & $100-200$ & Correo \\
Ferrocarril (1857, F.C.Oeste) & $40-45$ & 405 (en 9 horas) & 26 pasajeros p/vagón 8 tns. p/ vagón \\
\hline
\end{tabular}

Fuente: Zalduendo, 1975, Cuadro 6.1(a), p.261.

Un hito fundamental en el transporte terrestre lo constituyó la introducción hacia mediados del siglo XIX de las galeras y diligencias norteamericanas, también conocidas como 'mensajerías' (Gesualdo, 1986). En la provincia de Buenos Aires luego de la caída de Rosas en 1852, el catalán Joaquín Fillol estableció una flota de diligencias, las Mensajerías Argentinas, con recorridos que partían hacia todo el territorio provincial (Jewell, 1966, p.16). En 1855, Fillol trasladó su empresa de la provincia de Buenos Aires a Rosario; allí, asociado con su compatriota Juan Rusiñol, fundó las Mensajerías Nacionales Iniciadoras, estableciendo por primera vez el servicio de galeras o diligencias entre Rosario y Córdoba, cuatro veces al mes, y un servicio especial entre Rosario y Mendoza, dos veces por mes. En 1858, el riojano Timoteo Gordillo (1814-1894) formó la empresa Mensajerías Argentinas, con una frecuencia de cuatro viajes al mes entre Rosario y Córdoba, dos viajes por mes entre Córdoba y Catamarca, un viaje por mes entre Córdoba y Tucumán y un viaje por mes entre Tucumán y Salta. Asimismo, al servicio de diligencias entre Buenos Aires y Rosario, Gordillo agregó dos viajes más que se efectuaban en combinación con los arribos y partidas a la primera de estas ciudades de los vapores de la compañía británica Royal Mail Steam Packet Company.

El avance que significaron las mensajerías en cuanto a velocidad, hacia la época de su introducción (1848-1852), fue notorio: como se ve en el Cuadro 2, utilizando el sistema de postas para mudar caballos, estos vehículos recorrían hasta treinta leguas diarias (unos 150km), contra las tres u ocho leguas diarias de las carretas (15 a 40km). Así, el viaje entre Rosario y Córdoba que demoraba entre 25 y treinta días utilizando estas últimas, se hacía en diligencias en tres o cinco; el trayecto entre Rosario y Mendoza que demandaba, dependiendo de la estación, entre 35 y sesenta días en carreta, se realizaba en diez o 15 en galera. 
Cuadro 2

Comparación de la duración de los viajes entre distintas ciudades en distintos medios de transporte

\begin{tabular}{lcc}
\hline Medios & Distancia & Días \\
\hline Carretas & Mendoza-Valparaíso & $10-20$ \\
& Mendoza-Buenos Aires & $30-60$ \\
& Rosario-Tucumán & 35 \\
& Rosario-Mendoza & $35-60$ \\
& Rosario-Córdoba & $25-30$ \\
& Buenos Aires-Salta & $60-70$ \\
\hline Carros pesados & Tucumán-Salta & 12 \\
\hline Diligencias & Rosario-Mendoza & $10-15$ \\
& Tucumán-Salta & 7 \\
& Tucumán-Santiago del Estero & 3 \\
& Córdoba-Santiago del Estero & $6-9$ \\
& Rosario-Santa Fe & $1-3$ \\
& Rosario-Córdoba & $3-4$ \\
& Buenos Aires-Mendoza & $10-12$ \\
\hline Caballo & Mendoza-San Juan & 2 \\
& Buenos Aires-Tandil & 6 \\
\hline Felerria & San Juan-Valparaíso & 5 \\
& Santa Fé-Córdoba & 4 \\
\hline Vapores & Buenos Aires-Mendoza & 15 \\
\hline & San Juan-Córdoba & $6-8$ \\
\hline
\end{tabular}

Fuente: Zalduendo, 1975, Cuadro 6.1(b), p.262.

De manera simultánea a la introducción de las diligencias y galeras, se dio la incorporación de los primeros vapores y barcazas a motor en los ríos Paraná y Uruguay. Desde fines de la década de 1830, el tráfico fluvial era realizado por pequeñas flotas en manos de europeos, en su mayoría sardos y genoveses, utilizando goletas, sumacas, pailebotes, cúters y chalupas; al igual que en Estados Unidos, es en el transporte fluvial donde primero se aplicó el vapor, más precisamente hacia fines de la década de 1840 (Kroeber, 1957; Regalsky, Rosal, Schmit, 2001). Según Zalduendo (1975), los vapores significaron una extraordinaria revolución desde el punto de vista económico, ya que posibilitaron el desplazamiento de mayor cantidad de bienes y pasajeros en menor tiempo, con un costo reducido. En efecto, como se observa en el Cuadro 2, si los veleros tardaban veinte días en cubrir la ruta entre Buenos Aires y Rosario, los vapores hacían el mismo viaje en cuarenta horas (treinta, río abajo), cargando hasta trescientas toneladas y haciendo dos paradas en San Pedro y San Nicolás. En contraposición, el trayecto entre Buenos Aires y Asunción del Paraguay, que a inicios del siglo XIX demandaba entre dos y tres meses, se podía realizar en un vapor en solo diez días. Recordemos aquí que, a principios de 1827, el naturalista francés Alcide d'Orbigny empleó algo más de un mes en completar su viaje por 
el Paraná, desde Buenos Aires hasta Corrientes, utilizando embarcaciones movidas alternadamente por velas y remo (Podgorny et al., 2008).

Finalmente, para tener una idea comparativa a escala internacional, si en la primera mitad del siglo XIX con los buques de vela se tardaba de sesenta a setenta días en cubrir el trayecto entre los puertos de Europa occidental y América del Sur, utilizando los vapores de la compañía británica Royal Mail Steam Packet Company, hacia 1856, se demoraba alrededor de treinta días en unir el puerto de Southampton con Buenos Aires (Burmeister, 1943-1944, t.1, p.5-26).

\section{Los medios de transporte y locomoción en el desarrollo del viaje por los Estados del Plata del naturalista prusiano Hermann Burmeister}

Los avances en los medios de transporte y locomoción reseñados hasta aquí, introducidos hacia la segunda mitad de la década de 1850, tanto en el orden nacional como el internacional, coadyuvaron con el desarrollo de los viajes de exploración por las provincias de la confederación. Se sucedieron así los viajes del geógrafo y médico francés Víctor Martín de Moussy (1855-1858), el del mencionado naturalista alemán Hermann Burmeister (18571860), el de Charles Lina Beck-Bernard (1857-1862) por el Paraná, el del suizo J.J. von Tschudi (1858-1860) a las provincias del norte y el del médico italiano Paolo Mantegazza (1858 y 1861-1863), entre otros. El viaje que realizó el segundo de ellos da cuenta de la influencia del sistema de transportes, descrito anteriormente que combinaba carretas, vapores y diligencias, sobre las prácticas en el terreno del naturalista. Profesor de zoología en la Universidad de Halle desde 1837, Burmeister (1807-1892) recorrió entre 1857 y 1860 las provincias del interior del país, subvencionado por el gobierno imperial prusiano, con el objetivo de estudiar la fauna, la flora, y la climatología, y armar colecciones de zoología y geología para el gabinete universitario a su cargo. Al mismo tiempo, examinó detenidamente las formaciones geológicas cordillerana y terciaria del Paraná, permaneciendo para ello 14 meses en Mendoza y un año en Entre Ríos.

Con un pasaporte expedido por la Administración de Correos de la confederación, que lo habilitaba para utilizar el sistema de postas y para el transporte libre de cargas, y con un carretón cubierto, de dos ruedas, cedido por el gobierno, Burmeister partió de Rosario hacia Mendoza, tardando 13 días en llegar a destino (Burmeister, 1943-1944, t.1, p.114154). Si como ha sido señalado en los trabajos de historia económica, la marcha lenta de los medios de locomoción de entonces, animados por la tracción a sangre sobre rutas en malas condiciones de transitabilidad durante algunas estaciones del año, atentaba contra el desarrollo del comercio y la integración de los mercados regionales (Zalduendo, 1975), por el contrario, reportaba para el trabajo del naturalista algunas ventajas. Estas se derivaban precisamente de la característica principal de la tecnología del transporte movido por animales, como es su flexibilidad. Gracias a esta característica, la carreta o las diligencias podían adaptar su velocidad, sus paradas y el trayecto a recorrer de acuerdo a los deseos y necesidades de sus pasajeros, o a otras circunstancias del orden de lo contingente (Schivelbusch, 1988). En primer lugar, el viajero podía llevar un registro minucioso en sus libretas de las variaciones sucesivas y paulatinas en el ambiente y en la composición de la 
flora, la fauna y la gea en las diversas regiones, a lo largo de la ruta. Así, Burmeister pudo apreciar la distribución de especies de insectos y animales gracias a las observaciones tomadas en distintos puntos, muy alejados los unos de los otros, a lo largo del camino carretero que unía Rosario con Mendoza. ${ }^{1}$ En cuanto al armado de colecciones, la baja velocidad alcanzada permitía efectuar disparos a los animales con el carro en movimiento - haciendo que posteriormente el capataz o un ayudante recogiera el espécimen para prepararlo luego, operación que el ferrocarril dificultará, recolectar ejemplares botánicos sin necesidad de detener la marcha y realizar observaciones sobre los hábitos de la fauna. ${ }^{2}$

Asimismo, las muy frecuentes demoras en las postas, esperando nuevos animales para hacer la muda de caballos, constituían oportunidades excepcionales para la recolección de especimenes, ya que todo el sistema de postas estaba construido, por lo general, siguiendo la disponibilidad de aguadas, ríos o arroyos, y la presencia de pasturas para los animales y montes para la provisión de leña como combustible. Esta combinación de abundancia de agua y vegetación hacían de las postas y sus alrededores los puntos de recolección de insectos más interesantes. ${ }^{3} \mathrm{Al}$ mismo tiempo, las numerosas paradas que había que realizar para reparar los muy habituales desperfectos en los vehículos, como la rotura de los ejes y correas, o el recambio de material rodante, hacía que se debiera permanecer en el lugar hasta que se enviara un 'chasque' de la posta más cercana con un nuevo carro o las piezas nuevas y herramientas para hacer las reparaciones; ese 'tiempo muerto' era aprovechado por Burmeister para aumentar con nuevas especies su colección entomológica. ${ }^{4}$

La mayor parte de estas ventajas se desvanecían si el viajero optaba por utilizar, en vez de una carreta, las más veloces diligencias o galeras cuyo objetivo, como ya se ha mencionado, era el de transportar pasajeros a la mayor velocidad posible. Como se puede apreciar en el Cuadro 1, si la carreta alcanzaba una velocidad de tres a cuatro kilómetros por hora, recorriendo en consecuencia de 15 a $20 \mathrm{~km}$ por día, las galeras o diligencias, en cambio, alcanzaban velocidades de hasta $20 \mathrm{~km}$ por hora, cubriendo así trayectos de hasta $150 \mathrm{~km}$ diarios. Adelantando así una de las características derivadas de la tecnología ferroviaria, de cierto modo esta ganancia de tiempo implicaba para el naturalista la imposibilidad de obtener información valiosa acerca de las especies vegetales o animales, o la composición general del paisaje, a lo largo del camino recorrido. Así, en el camino que unía Córdoba con la ciudad de Tucumán, si bien Burmeister pudo constatar mediante observaciones desde la pequeña ventanilla de la galera en que viajaba que el matorral en esas regiones estaba compuesto por las mismas especies que crecían en los campos dendríticos de los alrededores de Mendoza - como el chañar y el espinillo -, no pudo hacer una descripción acabada ni recolectar muestras para su colección de las nuevas variedades propias de esa región, precisamente por la alta velocidad que había alcanzado el vehículo. ${ }^{5}$

Otra limitación que presentaban las galeras y diligencias era que, al ser básicamente un transporte de pasajeros, tenían una capacidad de carga limitada: por este motivo, en el viaje de regreso de Mendoza a Rosario, si bien Burmeister optó por la diligencia como transporte personal, envió en una tropa, custodiando las colecciones formadas en la cordillera, a su asistente Anton Göring (1836-1897) con el objetivo adicional que "durante la marcha reuniera asiduamente más especimenes a fin de aumentar convenientemente las reservas de mi colección" (Burmeister, 1943-1944, t.1, p.359). 


\section{El acceso al terreno en la era del vapor (1870-1914): la expansión de la red ferroviaria por los territorios del interior y las exploraciones geológicas y topográficas de la Academia Nacional de Ciencias de Córdoba}

Si bien el ferrocarril fue introducido en la provincia de Buenos Aires hacia 1857, con la creación de la empresa de capitales mixtos Sociedad del Camino de Hierro de Buenos Aires al Oeste - posteriormente conocida como Ferrocarril del Oeste - (Schvarzer, Gómez, 2006), es hacia la década de 1880 que comenzó en Argentina una verdadera 'fiebre ferroviaria', ligada principalmente a la consolidación del modelo agroexportador. En efecto, si entre aquella fecha y 1880 sólo se construyeron en promedio $100 \mathrm{~km}$ de vías por año, en la década siguiente la extensión de la red cuadruplicó su tamaño, pasando de 2.432 a $9.397 \mathrm{~km}$ (Schickendantz, Rebuleto, 1994). Esta primera fase de expansión fue motorizada por el Estado, que ofreció beneficiosas garantías para los inversores extranjeros, en su mayor parte de origen británico. Los trabajos de construcción se concentraron en tres grandes áreas: en la campaña bonaerense, asociadas a la prosperidad de la ganadería lanar (Ferrocarril Oeste, desde 1857 y Ferrocarril Sud, desde 1867); en Santa Fe, ligadas al explosivo crecimiento agrícola impulsado por las colonias (Ferrocarril Central Argentino, desde 1870); y en las áreas del interior, asociadas con la producción de cultivos industriales como la caña de azúcar en Tucumán (Ferrocarril Central Norte, desde 1876) y la vid en Mendoza (Ferrocarril Andino, desde 1885). En consecuencia, durante la década de 1880 quedó definida la red ferroviaria nacional que unió las ciudades de Buenos Aires, La Plata, San Luis, Mendoza, San Juan, Rosario, Córdoba, Tucumán, Catamarca y Santiago del Estero; en la década siguiente se agregarán La Rioja, Salta, Jujuy, Corrientes y Paraná. Al cerrarse esta primera fase de expansión, hacia 1892, la longitud total de la red ferroviaria superaba los 13.500km (Schickendantz, Rebuleto, 1994; Roccatagliata, 1987). ${ }^{6}$

El crecimiento notable de la red impulsó el acceso a regiones hasta ese entonces remotas, habilitando en consecuencia la posibilidad de estudiarlas: cada nueva estación inaugurada se transformaba así en una nueva 'puerta de acceso' al terreno. Como ha señalado Vetter (2004) en su estudio de la expansión de las expediciones hacia el Oeste norteamericano, asociada a la ampliación del tendido de la red del Union Pacific, el impacto más obvio del ferrocarril sobre las expediciones fue del orden práctico: repentinamente fue más fácil, rápido y seguro trasladar equipamiento, personal y colecciones hacia y desde el campo. Otros cambios cualitativos importantes que introdujo el ferrocarril, según el mismo autor, fueron la concentración de los trabajos de investigación a lo largo de las vías férreas, desplazando asî a las vías fluviales o los viejos caminos carreteros, y la posibilidad de alcanzar los sitios en poco tiempo, lo que desalentó las exploraciones realizadas hasta entonces a lo largo de toda la ruta. En el caso del río de la Plata, podemos observar que este último tipo de exploraciones había dominado el período anterior a la introducción del ferrocarril y los barcos a vapor y se había caracterizado por el estudio detenido del paisaje a lo largo de todo el trayecto determinado por la ruta y por la permanencia de largo tiempo en puntos acotados de interés para el viajero como en los ejemplos citados anteriormente de Alcide d'Orbigny (1827), Charles Darwin (1833) y Hermann Burmeister (1857-1860).

En cuanto al viaje de acceso al terreno, el punto de pasaje entre la utilización de este sistema de transporte vertebrado alrededor de las postas y vehículos de tracción a sangre al 
dependiente de una red de estaciones unidas por locomotoras a vapor, puede ilustrarse con el caso de los trabajos de relevamiento topográfico y geológico llevados a cabo por los científicos alemanes de la Academia Nacional de Ciencias de Córdoba en los pocos años que median entre su fundación, en 1870, y el lapso que va de 1875 a 1886, período en el cual se registró la extensión de las vías férreas tanto hacia la región del norte como hacia Cuyo. ${ }^{7}$ En su primer viaje de estudio de 1871, el doctor Alfred Wilhelm Stelzner (1840-1895) - profesor alemán de mineralogía y geología contratado por Burmeister que trabajó en esa institución entre el 6 de abril de 1871 y el 2 de junio de 1874 - reconoció el norte de las Sierra de Córdoba y atravesó en sus principales perfiles las cadenas montañosas de las provincias de Tucumán, Catamarca y La Rioja, utilizando como base el mapa levantado por Martín de Moussy y empleando para completar su itinerario casi cinco meses (Stelzner, 1966; Pastore, 1925). Tanto el recorrido y los tiempos de viaje, determinados ambos por el sistema de los correos de posta, las carretas, caballos y mulares, no difirieron en lo sustancial con el realizado por Burmeister hacia fines de la década de 1850, debiendo pasar primero por Santiago del Estero para llegar hasta Tucumán y las provincias del norte. Así, partiendo de Córdoba, Stelzner demoró casi veinte días en llegar a aquella ciudad; desde allí se dirigió a la provincia de Catamarca, a lomo de mula, por el valle de Santa María, para estudiar los establecimientos mineros con sede en las sierras graníticas y traquíticas de Capillitas y Atajo, continuando el recorrido por Belén, Tinogasta, Fiambalá, y de allí hacia el sur, bordeando la sierras de Tinogasta, para estudiar las minas de Famatina. Regresó desde allí a la ciudad de Córdoba, en siete días, utilizando el servicio de diligencias (Stelzner, 1873, p.584-585). En ese viaje de cinco meses, pudo reconocer la existencia de la formación terciaria en las proximidades de San José, en el valle de Santa María (Catamarca) - hasta entonces desconocida para esos parajes - y recoger petrificaciones en la sierra de los Angulos (La Rioja). Asimismo, las limitaciones en el transporte y la logística no le permitieron armar, como pretendía en un principio, una colección de rocas amplia y representativa de la geología de aquellas regiones (Stelzner, 1873, p.588-589). En su segundo viaje del año 1872-1873 recorrió las sierras de Córdoba y San Juan, de allí pasó a Chile, regresando por Mendoza y San Juan, empleando nuevamente casi cinco meses. A pesar de haber planeado un viaje para el verano de 1873-1874, Stelzner no pudo visitar las sierras de San Luis por falta de mulas, debiendo conformarse con una nueva excursión por las sierras de Córdoba en un lapso de tres semanas y cubriendo una zona algo más extensa que en su viaje anterior. Con los datos, las observaciones y las colecciones de minerales reunidas en esos viajes, Stelzner publicó primero el trabajo "Comunicaciones sobre la geología y mineralogía de la República Argentina", en los Anales de Agricultura (1873) y en el primer tomo de las Actas de la Academia Nacional de Ciencias. Posteriormente publicó, ya en Alemania, su obra Beiträge zur Geologie und Paleontologie der Argentinischen Republik auf anordnung der Argentinischen National-Regierung (1876 y 1885) con la colaboración de Hans Bruno Geinitz, Immanuel Kayser y Carl Gottsche. Los mapas geológicos anexos a esas obras nos dan una idea aproximada de la zona que pudo recorrer detenidamente, en sus mínimos detalles, limitada principalmente al sistema serrano de Córdoba.

El doctor Ludwig Brackebusch (1849-1906), su sucesor en el cargo entre 1874 y 1891, pudo aprovechar las ventajas que trajo aparejadas el avance del ferrocarril por las provincias del interior. El objetivo principal de las exploraciones llevadas a cabo por Brackebusch se 
centró en la obtención de datos topográficos con el fin de hacer relativamente más precisa la ubicación de sus observaciones geológicas, las que se extendieron ciertamente a todas aquellas regiones del norte y el centro del país, como San Luis, Salta y Jujuy, que no había podido visitar Stelzner por dificultades de logística (Kühn, 1916; Pastore, 1925). Dada la particular condición de la geología de amplias zonas de la región noroeste - caracterizadas por su uniformidad de aspecto y composición -, la introducción del ferrocarril posibilitó la confección de mapas geológico-topográficos que representaban áreas mucho más vastas que las estudiadas originalmente por Stelzner, haciendo posible, en primer lugar, la toma de muestras de manera bastante rápida en puntos seleccionados y muy distantes unos de otros; en segundo lugar, permitiendo calcular y registrar las mediciones astronómicas de posición tomando como base las posiciones calculadas por los ingenieros para el tendido de las líneas férreas; y, en tercer lugar, facilitando el envío de las pesadas colecciones de rocas a Córdoba a medida que se las iba armando en los distintos puntos de recolección y observación. De ese modo, durante sus primeros años en Córdoba, Brackebusch pudo proyectar la organización de sus expediciones siguiendo el avance de las secciones del ramal ferroviario que uniría esa capital con Cuyo y las extensiones hacia Catamarca, Salta y Jujuy. ${ }^{8}$ Con lo datos obtenidos en todos estos viajes, Brackebusch publicó en 1882 la carta de Jujuy (1:1.000.000) (Brackebusch, 1883); en 1889, la Carta de la República Argentina (1:500.000) presentada en la Exposición Internacional de París de ese año en relieve tallado en madera sobre 72 planchas conformando, desplegadas, una superficie total de 36 metros cuadrados; en 1891, su célebre Mapa de la República Argentina (1:1.000.000); y en 1892, el Mapa geológico del interior de la República Argentina (1:1.000.000) (Brackebusch, 1966).

\section{La expansión de la red ferroviaria y las exploraciones botánicas y zoológicas de la Academia Nacional de Ciencias de Córdoba}

A pesar de la mayor velocidad en el acceso al terreno provista por el ferrocarril y de su utilidad para los estudios de geología y topografía descritos en el apartado anterior, es dable considerar aquí la implicancia de aquella en el desarrollo de las expediciones de los miembros de la Academia Nacional de Ciencias que estaban especializados en otras ramas de las ciencias naturales, como la botánica y la zoología, cuyo trabajo en el terreno revestía otras características. Así, para el estudioso dedicado a la botánica, la velocidad no constituía una condición necesaria para los trabajos de herborización en el terreno. Más bien todo lo contrario. Como señaló el botánico alemán de la Academia Nacional de Ciencias, Paul Günther Lorentz (1835-1881), contrastando con la uniformidad de las formaciones geológicas del norte, la abundancia y riqueza de especies vegetales en esas regiones hacía que el medio de transporte ideal fuera el carro tirado por caballos, ya que, debido a su flexibilidad, ofrecía la posibilidad de detener la marcha cuando el estudioso lo deseaba. ${ }^{9}$ Además de la autonomía de movimientos que proporcionaba, otra ventaja que traía aparejada el carro era su capacidad de carga y el espacio para transportar y desplegar los elementos necesarios para trabajar en la preparación de las plantas - como los grandes rollos de papel secante, las prensas y las soluciones químicas - o para el almacenaje de los especimenes ya preparados para su conservación y estudio posterior. ${ }^{10}$ 
Por su parte, el zoólogo holandés de la Academia Nacional de Ciencias, Hendrik Weyenbergh (1842-1885), señaló que, comparativamente y por las características propias de su objeto de estudio, el viaje de estudios del zoólogo obligaba a la realización de exploraciones de carácter intensivo en puntos geográficos muy acotados, debiendo el estudioso permanecer por mucho más tiempo en una región, más que explorar a lo largo de toda una ruta para cubrir un gran territorio como en el caso descrito anteriormente. ${ }^{11}$ Así, Weyenbergh concentró sus trabajos en los ríos e islas del Paraná y alrededores de Santa Fé, puntos que, como hemos visto, constituían el nodo central que estaba unido a Córdoba por el Ferrocarril Central Argentino y servido por una línea de vapores que cubría la ruta desde Rosario a Santa Fé y Paraná y que partía una vez a la semana. Ocupó así una semana en el viaje de ida y vuelta, combinando ferrocarril y vapor, y permaneció doce días explorando en el terreno (Weyenbergh, 1875-1878, p.220).

En la breve descripción que el estudioso holandés hizo de la fauna que habitaba en la región que media entre Córdoba y Rosario, ${ }^{12}$ podemos observar asimismo otra de las características que, según Schivelbusch, introdujo la tecnología ferroviaria, como es el 'viaje panorámico'. Este tipo de viaje, al cambiar la relación entre el viajero y el paisaje recorrido, modificó la percepción que se tenía de este último: la observación del paisaje recorrido se realizó a partir de ese momento desde la ventana del vagón, a lo largo de un recorrido lineal entre dos puntos determinados de antemano, y a gran velocidad (Schivelbusch, 1988, p.56). Así, la descripción de la fauna hecha por Weyenbergh, a partir de las observaciones realizadas en las horas que duraba el viaje en ferrocarril, contrasta con las realizadas por Burmeister en trayectos similares donde abundan los detalles acerca del hábitat y de las costumbres de los animales, así como las variaciones sucesivas en la distribución de las especies, observadas a lo largo de la ruta.

\section{La expansión del ferrocarril en las provincias del noroeste y el desarrollo de las exploraciones arqueológicas organizadas por las instituciones científicas de Buenos Aires y La Plata}

Como se desprende de lo expuesto hasta aquí, una consecuencia importante de esta rapidez en el acceso facilitada por la tecnología ferroviaria fue el establecimiento de una relación más estrecha y fluida de las instituciones científicas con sede en los centros urbanos y los sitios a investigar. Dada la particular configuración territorial que adquirió el tendido de la red en la Argentina, asociada a la consolidación del modelo económico agroexportador, se hace evidente la facilidad en el acceso al terreno que ofreció el ferrocarril para aquellas instituciones científicas ubicadas en la capital del país y sus zonas de influencia. En efecto, el trazado de la red troncal nacional siguió, en líneas generales, de forma paralela a las antiguas rutas de carretas y galeras. Una vez consolidada, la red provocó una reorganización del espacio que dejó de depender como eje articulador de las grandes vías fluviales, como el Paraná, Paraguay y río de la Plata, revalorizándose los ejes de comunicación hacia el interior del país (Roccatagliata, 1987; Regalsky, Rosal, Schmit, 2001). La consecuencia más inmediata de esto fue la reducción sustancial del tiempo de acceso a esas regiones. En relación con esto, recordemos aquí que hacia mediados del siglo XIX para trasladarse 
desde la ciudad de Buenos Aires a las provincias del norte se demoraba entre 25 y 35 días. ${ }^{13}$ Durante los primeros años que siguieron al establecimiento de los Ferrocarriles Central Argentino y Central Norte, para hacer el mencionado trayecto, el puerto de Rosario siguió siendo un punto de pasaje obligatorio: hacia 1881-1882, combinando tres líneas de ferrocarril con el vapor Buenos Aires-Rosario, el tiempo total del viaje entre la ciudad porteña y Tucumán se había reducido a cuatro o cinco días. ${ }^{14}$ Más aun, con la inauguración en 1886 de la línea de conexión entre los ramales que hasta entonces partían desde las cabeceras situadas en Buenos Aires y en Rosario, el tiempo de viaje entre las ciudades emplazadas en la rivera del Plata y las provincias del norte se redujo a la mitad, es decir, a dos o tres días. Esta notoria reducción en el tiempo de viaje, sumada a la ventaja de que el tren era un medio de transporte más seguro, directo y con fletes de carga relativamente baratos, provocó un marcado aumento en la cantidad de exploraciones arqueológicas, notablemente a partir de la década de 1890, haciendo posible la acumulación en masa de colecciones de objetos muy frágiles como las grandes vasijas procedentes de los valles Calchaquíes que difícilmente hubieran resistido un viaje en carreta, en el trayecto de más de $1.000 \mathrm{~km}$ que las separaban de los centros de estudio y exhibición en Buenos Aires o La Plata.

Asociado a esto, es interesante observar cómo el proceso de extensión de las vías del Ferrocarril Central Norte entre Tucumán y Jujuy primero, y entre esta última y el límite con Bolivia después, es acompañado por un desplazamiento hacia el norte de los trabajos de exploración y excavación arqueológica. En efecto, cada nuevo tramo y estación habilitada se transformaba en una nueva 'puerta de acceso' a los sitios a excavar. Entre 1887 y 1895 el Museo de La Plata centró sus actividades de exploración en la zona del valle de Santa María y en los alrededores de Andalgalá (Catamarca), demorando menos de 48 horas en cubrir el trayecto entre La Plata y Catamarca por medio de la nueva conexión que pasaba por Luján, Pergamino, San Nicolás y Córdoba, y el ramal del Central Norte que partía desde la estación Recreo hasta Chumbicha, en el sur de la provincia de Catamarca, desde donde se combinaba en la punta de rieles con la mensajería que se dirigía a la ciudad capital de San Fernando del Valle de Catamarca.

Con la extensión de las obras desde Tucumán hacia Salta, a principios de la década de 1890, Carlos V. Burmeister, naturalista viajero del Museo Nacional de Buenos Aires, exploró en Incahuasi-Seclantás, y, en 1895 Juan Bautista Ambrosetti (1865-1917) - a la sazón bibliotecario y director del Museo Arqueológico y Etnográfico del Instituto Geográfico Argentino - llegó desde Buenos Aires a Salta en tres días para dar inicio a la exploración de las grutas pintadas en los alrededores del establecimiento del doctor Indalecio Gómez, situado en Pampa Grande, tomando como punto de acceso a los yacimientos la estación Tala, posteriormente llamada Ruiz de los Llanos. Ambrosetti continuará los trabajos de excavación en las proximidades de la finca de Gómez hasta 1905 (Ambrosetti, 1906) y, en 1906, comenzó las exploraciones en la ciudadela de La Paya, donde el punto de acceso fue la estación Zuviría (a 1.719km de la ciudad de Buenos Aires), cuarta estación de un ramal por ese entonces recién habilitado que partía desde la ciudad de Salta hacia el sur por el valle de Lerma (Ambrosetti, 1907).

Por otro lado, desde 1906 el tren arribó a la localidad de Humahuaca (Jujuy) y al año siguiente se inició la construcción de las vías entre el pueblo de Tilcara y la frontera con 
Bolivia, adonde arribaría finalmente en 1910. En 1908, debido al envío de piezas por parte de su cuñado que trabajaba en la provincia de Jujuy, Ambrosetti comenzó las investigaciones en esa región; viajando desde Buenos Aires en tren, en compañía de su familia, en el trayecto que partía de Jujuy hacia el norte, y gracias a la vista panorámica obtenida desde la ventanilla del vagón, pudo identificar en los faldeos y en las alturas de los cerrillos, los cementerios y ruinas de la localidad de Tilcara (ubicada a $1.784 \mathrm{~km}$ de la ciudad de Buenos Aires) donde trabajaría en los años subsiguientes (Cáceres Freyre, 1963, p.81-83).

Por último, merece destacarse aquí otro aspecto relacionado con el proceso de construcción de las líneas férreas y los trabajos de exploración científica: las remociones de grandes volúmenes de tierra, para la construcción de obras como terraplenes, puentes, túneles y estaciones, constituyeron oportunidades excepcionales para realizar estudios geológicos, paleontológicos y arqueológicos. Como ha demostrado Michael Freeman (2001) las excavaciones, apuntalamiento de túneles, cortes de elevaciones de carácter montañoso y obras de diferente magnitud en los terrenos por donde debía pasar el ferrocarril, influyeron activamente en el avance del conocimiento geológico en Gran Bretaña. En el caso de la arqueología del noroeste argentino, podemos observar que los trabajos de ampliación de la línea entre Tilcara y Bolivia dejaron al descubierto numerosas sepulturas que posteriormente fueron 'saqueadas' por coleccionistas locales interesados en su venta (Boman, 1908, t.2, p.789).

\section{Consideraciones finales}

Como se ha visto en las páginas precedentes, la introducción de los medios de transporte a vapor en el río de la Plata produjo cambios referidos tanto a las formas de acceso al terreno disponibles al estudioso como a la organización del trabajo, el transporte de colecciones y el traslado de personal. Si bien los ferrocarriles y los vapores no desplazan totalmente a los medios de tracción a sangre que dominaron la etapa anterior - más bien se complementan entre sí -, se puede observar que la expansión de las redes por donde circulan los mismos introdujeron cambios en la organización de los itinerarios. Si hasta mediados del siglo XIX predominaron las exploraciones a lo largo del camino recorrido y la permanencia por tiempos prolongados en puntos específicos - como lo demuestran los itinerarios seguidos en los viajes de Alcide d'Orbigny (1827), Charles Darwin (1833) y Hermann Burmeister (1857-1860) -, posteriormente se puede observar que la relación más estrecha entre las instituciones científicas y las regiones a explorar, motorizada por el ferrocarril y los vapores, flexibilizaron notablemente el acceso a las mismas, facilitando el transporte de colecciones y acortando los tiempos de duración de los viajes. En ese sentido, en el caso de las exploraciones organizadas desde la Academia Nacional de Ciencias, se puede observar comparativamente las ventajas que introdujo el ferrocarril, permitiendo el acceso a regiones antes vedadas a los estudios geológicos, facilitando el traslado de muestras de minerales y rocas y brindando la posibilidad de realizar gran cantidad de registros de mediciones en un territorio amplio, coadyuvando así con el desarrollo de trabajos topográficos. En el caso de las expediciones de los académicos que se dedicaban a la botánica o la zoología, las ventajas se tradujeron fundamentalmente en una mayor flexibilidad en 
el acceso a las regiones a estudiar. Esta última ventaja fue importante también para las expediciones arqueológicas a la región noroeste, organizadas desde instituciones de la ciudad de Buenos Aires, donde se puede observar cómo la ampliación de los ramales hacia el límite con Bolivia se vio acompañada por el desarrollo progresivo de expediciones a las nuevas regiones que el ferrocarril atravesaba, facilitando al mismo tiempo el traslado de piezas delicadas como las vasijas cerámicas calchaquíes.

Por último, si bien excede con creces el período temporal abordado en este trabajo, merece destacarse que el ferrocarril, como medio de acceso a sitios lejanos de las ciudades de La Plata o Buenos Aires, parece haber sido el medio de transporte preferido por los investigadores al menos hasta mediados de la década de 1940. Esto puede deberse, sobre todo, a su baratura, sus ventajas en velocidad y facilidad de transporte de colecciones y por las tarifas promocionales en pasajes establecidas por las compañías estatales para los profesores universitarios. ${ }^{15}$

\section{AGRADECIMIENTOS}

Agradezco a Irina Podgorny y a un evaluador anónimo por las sugerencias y lectura crítica de este trabajo. El mismo fue realizado con el apoyo de una beca doctoral del Conicet y en el marco de los proyectos de investigación El Mapa de una Controversia Científica: Los Debates sobre la Antigüedad de la Formación Pampeana, 1880-1925 (PIP N.5675), y Museos, Instrumentos y Prácticas Científicas: Hacia un Estudio de la Cultura Material y de los Medios Técnicos de las Ciencias Naturales en la Argentina (PICT 2005 ET 3221), dirigidos por Irina Podgorny.

\section{NOTAS}

1 "Mientras se cambiaban los caballos en la estación, que se encuentra al norte poco distante del camino, buscando insectos, encontré la primera Nyctelia (Aulodera gibba Blanch. Voyage de D'Orbigny), coleóptero que pertenece a la zona cordillerana y que por su presencia en este lugar me demostraba que la influencia de aquélla ya se hace presente en el carácter orgánico del país" (Burmeister, 1943-1944, t.1, p.164).

2 "En todo el camino sólo vi pampas lisas, incultas y sin matorrales, sin ningún nuevo objeto que atrajera mi atención, salvo aquella bonita flor blanca, especie de Echites ... . Entre Tambito y Chucul, la vimos en varios sitios, pero siempre aislada entre el pasto; mi capataz me alcanzó una del suelo para verla con más detención."; "Un poco más allá observé también junto al camino al Eudinopus dytiscoides ... . Bajé del carretón para atraparlo, pero por sus movimientos no pude descubrir para qué le sirven a este animal las patas del medio tan extremadamente largas" (Burmeister, 1943-1944, t.1, p.152, 159-160).

3 "La posta [Médano de Gauna] se halla junto a una ciénaga, es decir al lado de un pequeño bajo húmedo en el que crecen juncos, sin tener otra cosa que invite a quedarse, aunque fue para mí de gran interés, porque durante la parada atrapé una cantidad de los más hermosos coleópteros, que pertenecen a los más notables de mi colección." (Burmeister, 1943-1944, t.1, p.178).

4 "El primero de mayo alcanzamos Esquina de Bustos, pero muy tarde, porque hubo varios desperfectos en los correajes. Poco después, volvió a quebrarse algo del carro de los equipajes, cuya reparación duró más de una hora, y más adelante se salió una rueda del eje, causa de una nueva demora. Entre tanto coleccionaba insectos, y encontré varias especies muy bonitas y nuevas para mí". (Burmeister, 1943-1944, t.1, p.364).

5 "Me hubiera agradado examinar estas plantas con más detención, pero la premura que llevaba la galera no me dio tiempo para esto; me contenté con conseguir, por lo menos, los frutos del quebracho". (Burmeister, 1943-1944, t.1, p.113). Posteriormente, en un viaje más detenido por la misma región, pudo establecer que se trataba del vinal y del guayacán.

${ }^{6}$ La segunda fase se dio entre 1900 y 1914, período donde se alcanzó el máximo nivel histórico de afluencia de inversiones extranjeras y de construcciones ferroviarias. Así, si en 1900 la longitud de la red era de $16.500 \mathrm{~km}, 14$ años más tarde la misma pasó a ser de $35.586 \mathrm{~km}$, con el $85 \%$ del negocio en manos 
de compañías privadas. La región pampeana, más específicamente la provincia de Buenos Aires, fue la receptora privilegiada de las inversiones, acompañando al proceso de modernización de la agricultura y de la ganadería bovina (Regalsky, 1989). Por otro lado, en esa etapa se establecieron también los primeros ramales de cierta envergadura en la Patagonia, hasta entonces comunicada con la capital federal por medio de los vapores.

${ }^{7}$ Acerca de la historia de la Academia Nacional de Ciencias de Córdoba, especialmente durante sus años iniciales, véase Tognetti, Page, Barbieri, 2000; Tognetti, Barbieri, 2004.

${ }^{8}$ En efecto, en el verano de 1875 estableció como base para el depósito de las colecciones geológicas a la ciudad de San Luis, debido a que "se ofrecía allá las mejores ocasiones para transportarlas a Córdoba por medio del Ferrocarril Trasandino" (Brackebusch, 1875-1878, p.189). Las secciones que se inauguraron por ese entonces, en orden cronológico, fueron las siguientes: Villa María-río Cuarto, $132 \mathrm{~km}$ (1873); río Cuartorío Quinto, 122km (1875); río Quinto-San Luis, 95km (1880); San Luis-La Paz, 120km (1883); La PazMendoza, 128km (1884); Mendoza-San Juan, 154km (1885) (cf. Zalduendo, 1975, p.410, nota 151).

9 "El Botánico puede esperar encontrar en todas partes, en la llanura como en las Sierras, objetos de su estudio. Para él es preciso viajar con toda independencia y la posibilidad de pararse a cada momento para juntar las plantas que se ofrecen y prepararlas en tiempo conveniente. Con este motivo hice construir un carro sencillo de dos ruedas, adaptado a los fines de mi viaje, contando al mismo tiempo, que los gastos de tal carro se compensarían en futuros viajes, ahorrándose así los gastos de la Mensajería para mí, mi sirviente y los baúles numerosos que un botánico se ve precisado a llevar consigo. Viajamos despacio y en gran parte a pie para observar mejor los objetos naturales que se ofrecieron, parándonos cuando entraba el sol, donde siempre hemos encontrado en el campo un poco de agua y pasto para los animales ... . Los objetos de botánica son mucho más numerosos que los de la Mineralogía y se encuentran casi en todas partes. Mientras las llanuras inmensas de esta República no ofrecen nada o casi nada al minerólogo [sic], a cada paso puede ofrecer al botánico algo que es digno de observación y en muchos casos de conservación y preparación, mientras las sierras de este país con su grandiosa uniformidad, solamente en pocos puntos ofrecen minerales o petrificaciones de interés para el sabio minerólogo, están cubiertas de una variedad de plantas, que ni yo, ni mi sucesor, ni el sucesor de él, jamás agotarán y que ofrecen objetos para estudios históricos todavía por siglos" (Lorentz, 1873, p.571-572, 581-582).

10 "los objetos botánicos requieren mucho más trabajo y aparatos que los mineralógicos. Cada planta precisa ser preparada y aprensada. Después es necesario cambiar el papel entre las plantas cada día, hasta que estén muy secas, lo que dura dos o tres semanas, y secar el papel húmedo al sol o al fuego. Por eso el botánico precisa en el viaje mucha más independencia en sus movimientos y muchos más baúles, especialmente a lo menos dos personas y una cantidad de papel secante. Por este motivo me hice construir como ya he indicado un carro para la llanura y en la sierra precisé a lo menos dos, y a veces tres cargas" (Lorentz, 1873, p.582).

11 "Los viajes zoológicos tienen una particularidad comparativamente a los de otros ramos. Las plantas y los minerales, por ejemplo, no se ocultan, no disparan a la vista del hombre: el botánico y el minerálogo[sic] saben donde hay que encontrarlos, y, estando una vez en el lugar indicado, pueden tomar tantos cuantos deseen; los animales, al contrario, se ocultan, huyen, escapan a la vista del observador, y de esta coincidencia resulta que aun en los parajes donde se sabe que tal o cual especie se encuentra, muchas veces no se la ve, porque se aleja de su perseguidor, burlándose de él, de sus esfuerzos y de sus armas. Por esta razón, en los viajes zoológicos se precisa, para dar una idea más o menos completa de la fauna del paraje que se visita, una permanencia más larga en la parte del país que se quiere estudiar. Cuanto más extenso es el territorio, tanto menos completo e importante será el resultado, especialmente respecto a las especies biológicas: quiero decir que, para el estudio, es mejor quedarse cazando quince días en un lugar, que atravesar en el mismo lapso toda una provincia; siendo el tiempo disponible igual, el resultado será en relación inversa a la extensión del paraje. Aquí se ve comprobada de una manera particular la verdad del proverbio francés 'qui trop embrasse, mal étreint'” (Weyenbergh, 1875-1878, p.219).

12 "Paso en silencio el viaje de Córdoba al Rosario, porque ofrece muy poco a la observación del zoólogo: en la pampa que atraviesa el ferrocarril se ven ordinariamente algunos avestruces (Rhea americana), algunas gamas (Cervus campestris) y caranchos (Polyborus vulgaris); he visto un solo zorro (Pseudolopex azarae); hay generalmente cantidades de langostas (Acridium paranense) y lechuzas (Strix curricularia); las últimas casi siempre con una posición e inmovilidad, que parecen individuos armados para un museo, sea al borde de las cuevas de las vizcachas, sea en las líneas del telégrafo" (Weyenbergh, 1875-1878, p.220).

${ }^{13}$ El trayecto obligado era partir en vapor hacia Rosario desde el puerto de las Conchas (hoy Tigre), por el río Paraná, haciendo dos paradas en San Pedro y San Nicolás. Del puerto santafesino se viajaba en las 
diligencias de Rusiñol y Fillol hasta Córdoba y de ésta, utilizando el mismo servicio, hacia Santiago del Estero, Tucumán, Salta y Jujuy (Von Tschudi, 1966).

${ }^{14}$ Así, el naturalista británico Ernest William White en 1881 partió desde la ciudad de Buenos Aires en ferrocarril y llegó en dos horas al puerto de Campana, sobre el río Paraná, donde abordó el vapor Proveedor que viajaba toda la noche para amarrar en Rosario a las 10 a.m. del día siguiente. El puerto rosarino estaba conectado entonces con la terminal del Ferrocarril Central Argentino, que partía a Córdoba a las 6 a.m. del día siguiente. Luego de un viaje de 15 horas hasta la capital mediterránea se llegaba a la estación terminal del Central Argentino que en ese punto estaba unida a la del Central Norte. El sistema estaba arreglado de manera tal que, a la hora y media de su arribo, el pasajero pudiera abordar el tren de trocha angosta a Tucumán (White, 1881-1882, t.1, p.204-218, 241-258; t.2, p.41-42).

${ }^{15}$ Hacia mediados de la década de 1940 los Ferrocarriles del Estado otorgaban franquicias especiales para los profesores universitarios, por un máximo de 60 días, para todas aquellas líneas que partían desde la ciudad de Córdoba, por medio de un abono en coche cama a precios muy reducidos (Archivo Histórico del Museo de La Plata, Sección Archivo del Instituto del Museo de la Plata, carpeta 69 (1945-1947), expediente letra E, n.1, año 1945).

\section{REFERENCIAS}

AMBROSETTI, Juan B.

Exploraciones arqueológicas en la ciudad prehistórica de La Paya (valle Calchaquí, provincia de Salta): campañas de 1906 y 1907. Buenos Aires: Facultad de Filosofía y Letras. (Publicaciones de la Sección Antropológica). 1907.

AMBROSETTI, Juan B.

Exploraciones arqueológicas en la Pampa Grande (provincia de Salta). Buenos Aires: Félix Lajouane. 1906.

BARBA, Enrique.

Rastrilladas, huellas y caminos. La Plata: Editorial Raigal. (Colección Campo Argentino).1956.

BOMAN, Eric.

Antiquités de la région andine de la République Argentine et du désert d'Atacama. Paris: Imprimerie Nationale. 1908.

BOSE, Walter B.L.

Historia de las comunicaciones en la República Argentina (Antecedentes y período 1862-1930). In: Academia Nacional de la Historia (Ed).

Historia argentina contemporánea. v.2, sección 2. Buenos Aires: El Ateneo. p.160-212. 1966.

BRACKEBUSCH, Ludwig.

Viaje en las Cordilleras de la República

Argentina, 1891. Boletín de la Academia

Nacional de Ciencias de Córdoba, Córdoba, t.45, p.197-223. 1966.

BRACKEBUSCH, Ludwig.

Viaje a la provincia de Jujuy. Boletín del Instituto Geográfico Argentino, Buenos Aires, t.4, p.9-17, 203-210, 217-226. 1883.

BRACKEBUSCH, Ludwig.

Informe sobre un viaje geológico hecho en el verano del año 1875 por las sierras de Córdoba y de San Luis. Boletín de la Academia Nacional de Ciencias de Córdoba, Córdoba, t.2, p.167-216. 1875-1878.

BURMEISTER, Hermann.

Viaje por los Estados del Plata, con referencia especial a la constitución física y al estado de cultura de la República Argentina (1857-1860). Buenos Aires: Mercur-Unión Germánica de la Argentina. 1943-1944.

CÁCERES FREYRE, Julián.

Juan B. Ambrosetti. Buenos Aires: Ediciones Culturales Argentinas. 1963.

CAMERINI, Jane.

Remains of the day: early Victorians in the field. In: Bernard Lightman (Ed.) Victorian science in context. Chicago: University of Chicago Press. p.354-377. 1997.

CAMERINI, Jane.

Wallace in the Field. Osiris, Chicago, v.11, p.44-65. 1996.

CÁRCANO, Ramón J.

Historia de los medios de comunicación y transportes en la Argentina. Buenos Aires: Félix Lajouane. 1893.

CASTRO ESTEVEZ, Ramón.

Historia de los correos y telégrafos de la República Argentina. Buenos Aires: Ed. Oficial de la Dirección General de Correos y Telégrafos. 1934-1942.

FREEMAN, Michael.

Tracks to a new world: railway excavation and the extension of geological knowledge in mid-nineteenth-century Britain. British Journal for the History of Science, London, v.34, p.51-65. 2001. 
GESUALDO, Vicente.

Todo sobre ruedas: carrozas, diligencias, galeras, sopandas y carretas. Todo Es Historia, Buenos Aires, n.227, p.8-31. 1986.

GOLDSTEIN, Daniel.

'Yours for science': the Smithsonian institution's correspondents and the shape of scientific community in nineteenth-century America. Isis, Chicago, v.85, p.573-599. 1994.

JEWELL, Carlos.

Mensajerías argentinas. Buenos Aires: Emecé. 1966.

KOHLER, Robert E.

Landscapes and labscapes: exploring the labfield border in biology. Chicago: University of Chicago Press. 2002.

\section{KROEBER, Clifton.}

The growth of the shipping industry in the río de la Plata region, 1794-1860. Madison: The University of Wisconsin Press. 1957.

KÜHN, Franz.

Deutsche geographische Arbeit in und über Argentinien. Zeitschrift der deutschen wissenschaftlichen Vereins sur Kultur und Landeskunde Argentiniens, Buenos Aires, n.5-6, p.261-75, 286-310. 1916

KUKLICK, Henrika; KOHLER, Robert. Introduction. Osiris, Chicago, v.11, p.1-16. 1996.

\section{LORENTZ, Paul Günther.}

Informe del dr. Lorentz (catedrático de Botánica, Facultad de Ciencias Matemáticas y Físicas de Córdoba) al Ministro de Justicia, Culto e Instrucción Pública, dr. D. Nicolás Avellaneda. In: Ministerio de Justicia, Culto e Instrucción Pública. Memoria del Ministerio de Justicia, Culto e Instrucción Pública, 1872. Buenos Aires: Imprenta La Unión. p.571-582. 1873.

PASTORE, Franco.

Nuestra mineralogía y geología durante los últimos cincuenta años, 1872-1922. Buenos Aires: Coni. (Evolución de las Ciencias en la República Argentina, 6). 1925.

PODGORNY, Irina.

Bones and devices in the constitution of paleontology in Argentina at the end of nineteenth-century Argentina. Science in Context, Tel Aviv, v.18, n.2, p.249-283. 2005.

PODGORNY, Irina.

Ser todo y no ser nada: paleontología y trabajo de campo en la Patagonia a fines del siglo XIX. In: Visacovsky, Sergio; Guber, Rosana (Comp.). Historias y estilos de trabajo de campo en la Argentina. Buenos Aires: Editorial Antropofagia. p.31-77. 2002.
PODGORNY, Irina et al.

Las formaciones geológicas sudamericanas en los viajes de Charles Darwin y Alcide d'Orbigny: mapas geológicos, fósiles e itinerarios. Registros, Revista anual de Investigación del Centro de Estudios Históricos Arquitectónico-Urbanos, Mar del Plata, v.5, n.5, p.1-25. 2008.

PODGORNY, Irina; SCHÄFFNER, Wolfgang. 'La intención de observar abre los ojos': narraciones, datos y medios técnicos en las empresas humboldtianas del siglo XIX. Prismas, Revista de Historia Intelectual, Quilmes, n.4, p.217-27. 2000.

REGALSKY, Andrés.

Foreign capital, local interests and railway development in Argentina: French investments in railways, 1900-1914. Journal of Latin American Studies, London, v.21, n.3, p.425-52. 1989.

REGALSKY, Andrés; ROSAL, Miguel A.; SCHMIT, Roberto.

Los transportes. In: Academia Nacional de la Historia (Org.) Nueva historia de la nación argentina. La configuración de la República independiente (1810-c.1914). Tercera parte, v.6. Buenos Aires: Grupo Editorial Planeta. p.173-203. 2001.

ROCCATAGLIATA, Juan.

Los ferrocarriles en la Argentina: un enfoque geográfico. Buenos Aires: EUdeBA. 1987.

ROZWADOWSKI, Helen M.

Small world: forging a scientific maritime culture for oceanography. Isis, Chicago, v.87, p.409-429. 1996.

SCHICKENDANTZ, Emilio; REBULETO, Emilio. Los ferrocarriles en la Argentina, 1857-1910. Buenos Aires: Fundación del Museo Ferroviario. 1994.

SCHIVELBUSCH, Wolfgang. Storia dei viaggi in ferrovia. Torino: Giulio Einaudi editore. 1988.

SCHVARZER, Jorge; GÓMEZ, Teresita. La primera gran empresa de los argentinos: el Ferrocarril del Oeste (1854-1862). Buenos Aires: Fondo de Cultura Económica. 2006.

SECORD, Ann.

Corresponding interests: artisans and gentlemen in nineteenth-century natural history". British Journal for the History of Science, London, v.27, p.383-408. 1994.

STAR, Susan L.; GRIESEMER, James R. Institutional ecology, 'transaltions' and boundary objects: amateurs and professionals in Berkeley`s Museum of Vertebrate Zoology, 1907-39. Social Studies of Science, London, v.19, p.387-420. 1989. 
STELZNER, Alfred.

Comunicaciones al profesor H.B. Geinitz (18712). Boletín de la Academia Nacional de Ciencias de Córdoba, Córdoba, t.45, p.115-127. 1966.

STELZNER, Alfred.

Informe del catedrático de mineralogía, prof. Alfred Stelzner al Ministro de Justicia, Culto e Instrucción Pública, dr. D. Nicolás Avellaneda. In: Ministerio de Justicia, Culto e Instrucción Pública. Memoria del Ministerio de Justicia, Culto e Instrucción Pública, 1872. Buenos Aires: Imprenta La Unión. p.584-591. 1873.

TOGNETTI, Luis Alberto; BARBIERI, Santiago. La Academia Nacional de Ciencias en el siglo XIX: los naturalistas, publicaciones y exploraciones. Córdoba: Academia Nacional de Ciencias. 2004

TOGNETTI, Luis Alberto; PAGE, Carlos; BARBIERI, Santiago (Coord.).

La Academia Nacional de Ciencias: etapa fundacional - siglo XIX. Córdoba: Academia Nacional de Ciencias. 2000.

VETTER, Jeremy.

Science along the railroad: expanding field work in the US Central West. Annals of Science, London, v.61, p.187-211. 2004.

VON TSCHUDI, Johann J.

Viaje por las Cordilleras de los Andes de Sudamérica, de Córdoba a Cobija, en el año 1858. Boletín de la Academia Nacional de Ciencias de Córdoba, Córdoba, t.45, p.115-27. 1966.

WEYENBERGH, Hendrick.

Informe sobre una excursión zoológica a Santa Fe practicada en 1876. Boletín de la Academia Nacional de Ciencias de Córdoba, Córdoba, t.2, p.217-43. 1875-1878.

WHITE, Ernest W.

Cameos from the Silver Land, or the experiences of a young naturalist in the Argentine Republic. London: John van Voorst. 1881-1882.

ZALDUENDO, Eduardo A.

Libras y rieles: las inversiones británicas para el desarrollo de los ferrocarriles en la Argentina, Brasil, Canadá e India durante el siglo XIX. Buenos Aires: El Coloquio. 1975.

\section{$\rightarrow \rightarrow \rightarrow<<$}

